\title{
Evaluation et impact non clinique des dispositifs médicaux
}

Benoît Dervaux ${ }^{1}$, Karine Szwarcensztein ${ }^{2}$, Anne Josseran ${ }^{3}$ et les participants à la table ronde $N^{\circ} 4$ de Giens XXX: Alexandre Barna ${ }^{4}$, Cédric Carbonneil ${ }^{5}$, Karine Chevrie ${ }^{6}$, Frédérique Debroucker ${ }^{7}$, Anne Grumblat ${ }^{8}$, Olivier Grumel ${ }^{9}$, Jacques Massol ${ }^{10}$, Philippe Maugendre ${ }^{11}$, Hubert Méchin ${ }^{12}$, David Orlikowski ${ }^{13}$, Christophe Roussel ${ }^{14}$, Catherine Rumeau-Pichon ${ }^{15}$, Jean-Patrick Sales ${ }^{15}$ et Eric Vicaut ${ }^{16 \dagger}$

1 Faculté de Médecine, CHRU, Lille, France

2 Johnson \& Johnson Produits de Santé, Issy-les-Moulineaux, France

3 SNITEM, Courbevoie, France

4 CEDIT, Paris, France

5 Direction Générale de l'Organisation des Soins, Ministère des Affaires Sociales, de la Santé et des Droits des Femmes, Paris, France

6 EOS Imaging, Paris, France

7 Medtronic France, Boulogne-Billancourt, France

$8 \mathrm{CHU}$, Besançon, France

9 Baxter France, Maurepas, France

10 Institut Phisquare, Paris, France

11 Sanofi France, Paris, France

12 Helsia, Paris, France

13 Hôpital Universitaire Raymond Poincaré, Garches, France

14 3M France, Paris, France

15 Haute Autorité de Santé, Saint-Denis, France

16 Hôpitaux Universitaires Saint-Louis Lariboisière, Paris, France

Texte reçu le 5 janvier 2015 ; accepté le 6 janvier 2015

\section{Mots clés :}

dispositifs médicaux : évaluation ; Health Technology Assessment
Résumé - Les dispositifs médicaux (DM) couvrent une large variété de produits. Ils accompagnent, au gré des innovations technologiques, l'évolution des pratiques médicales. Les innovations dans le champ du DM peuvent améliorer les conditions d'utilisation de la technologie de santé et/ou modifier l'organisation des soins au-delà du strict bénéfice thérapeutique ou diagnostique pour le patient. Cependant, ces critères non purement cliniques semblent n'être que rarement documentés ou pris en compte dans l'évaluation des DM lors des décisions de remboursement au niveau national ou de référencement au niveau des établissements de santé alors même que des modèles d'évaluation multidimensionnelle des technologies de santé, prenant en compte le point de vue de l'ensemble des acteurs du système de santé, ont été élaborés. Dans cet article, après avoir rappelé le contexte de l'évaluation des technologies de santé en France, une définition des critères d'évaluation non clinique des DM est proposée et un arbre de décision pour l'évaluation des DM est décrit. Des pistes de réflexion sont proposées en conclusion.

Abréviations : voir en fin d'article.

\footnotetext{
$\dagger$ Les articles, analyses et propositions des Ateliers de Giens n’engagent que leurs auteurs et ne préjugent pas de la position de leur organisme de tutelle.
} 


\section{Introduction}

Le dispositif médical (DM) est défini dans le code de la Santé publique (article L5211-1) de la manière suivante : «On entend par dispositif médical tout instrument, appareil, équipement, matière, produit, à l'exception des produits d'origine humaine, ou autre article utilisé seul ou en association, y compris les accessoires et logiciels nécessaires au bon fonctionnement de celui-ci, destiné par le fabricant à être utilisé chez l'homme à des fins médicales et dont l'action principale voulue n'est pas obtenue par des moyens pharmacologiques ou immunologiques ni par métabolisme, mais dont la fonction peut être assistée par de tels moyens. Constitue également un dispositif médical le logiciel destiné par le fabricant à être utilisé spécifiquement à des fins diagnostiques ou thérapeutiques». Le code de la Santé publique définit aussi les dispositifs médicaux implantables actifs ainsi que les dispositifs médicaux de diagnostic in vitro ${ }^{1}$ qui entrent dans le champ de la réflexion menée ci-dessous.

Ainsi les DM couvrent une large variété de produits allant des consommables à l'équipement lourd d'imagerie, en passant par les prothèses implantables, les aides techniques pour personnes handicapées, les pansements, les orthèses, certains logiciels... On distingue classiquement trois grandes catégories de DM :

1. les matériels consommables ou implantables, à usage unique ou à usage individuel ;

2. le matériel réutilisable ;

3. 1'équipement.

Les DM se distinguent par plusieurs spécificités dont deux particulièrement à prendre en compte ici :

- un cycle de vie qui peut être très court : le rythme des évolutions technologiques dans ce secteur est important; les cycles d'innovation d'un produit donné sont d'environ 2 à 5 ans. De

\footnotetext{
1 Article L5211-1 du code de la Santé Publique : « Les dispositifs médicaux qui sont conçus pour être implantés en totalité ou en partie dans le corps humain ou placés dans un orifice naturel, et qui dépendent pour leur bon fonctionnement d'une source d'énergie électrique ou de toute source d'énergie autre que celle qui est générée directement par le corps humain ou la pesanteur, sont dénommés dispositifs médicaux implantables actifs. »

Article L5121-1 du code de la Santé Publique : «Constituent des dispositifs médicaux de diagnostic in vitro les produits, réactifs, matériaux, instruments et systèmes, leurs composants et accessoires, ainsi que les récipients pour échantillons, destinés spécifiquement à être utilisés in vitro, seuls ou en combinaison, dans l'examen d'échantillons provenant du corps humain, afin de fournir une information concernant un état physiologique ou pathologique, avéré ou potentiel, ou une anomalie congénitale, pour contrôler des mesures thérapeutiques, ou pour déterminer la sécurité d'un prélèvement d'éléments du corps humain ou sa compatibilité avec des receveurs potentiels. »
}

fait, il s'agit souvent d'évolutions technologiques graduelles qui peuvent apporter des modifications en termes de prise en charge diagnostique, thérapeutique ou de compensation du handicap;

- un caractère dit opérateur-dépendant : les DM sont associés à une utilisation par un soignant (chirurgien, médecin, infirmier...), le patient, ou quelqu'un de son entourage. De cette spécificité découle le principe de DM plus ou moins opérateurdépendant et l'idée de bénéfices au-delà du strict bénéfice thérapeutique (ou diagnostique) pour le patient.

Ainsi, les DM accompagnent, au gré des évolutions technologiques, l'évolution des pratiques médicales et peuvent jouer un rôle important dans l'organisation des soins (autonomie du patient, diminution de la charge en soins, accès aux soins, impact sur les durées d'hospitalisation, d'intervention chirurgicale...) ou dans la sécurité des soins (sécurité au travail des soignants, meilleure ergonomie...).

Ces critères non purement cliniques des DM semblent n'être que rarement documentés ou pris en compte lors de l'évaluation, que ce soit lors des évaluations au niveau national pour l'admission au remboursement (Commission Nationale d'Evaluation des Dispositifs Médicaux et des Technologies de Santé [CNEDiMTS], Commission d'Evaluation Economique et de Santé Publique [CEESP]) ou bien au niveau local lors des décisions d'adoption d'une nouvelle technologie dans un établissement de santé par exemple.

De ce constat que l'évaluation purement clinique ne résume souvent pas à elle seule l'effet d'un DM, a découlé la question de la pertinence de l'évaluation clinique seule et de son impact sur la prise de décision. Ainsi, l'objectif de la table ronde était 1- d'analyser dans quelle mesure les critères non cliniques étaient actuellement intégrés ou pas aux évaluations nationales et locales ; 2- s’il était légitime et pertinent d'intégrer ces critères dans les évaluations et si oui, de quelle manière, enfin; 3- si la prise en compte de ces critères dans les évaluations pouvait avoir un impact sur la prise de décision.

\section{Eléments de définition}

La finalité des DM n'est pas uniquement thérapeutique. Elle peut être diagnostique mais aussi de compensation du handicap. Dans ce domaine de la compensation du handicap, les DM visent à accroître l'autonomie et la qualité de vie des personnes. Par ailleurs, nombre de DM interviennent indirectement dans la prise en charge des patients. Ces dispositifs sont à destination des professionnels de santé et non des patients. Ils peuvent sécuriser les conditions de réalisation d'un acte ou d'un geste sans 
nécessairement avoir un impact immédiat ou aisément quantifiable sur la santé des patients. Par exemple :

- une meilleure ergonomie ayant un impact sur les utilisateurs (soignants, patients, aidants);

- une sécurisation des actes (prévention des accidents exposant au sang, diminution du risque chimique-ionisant);

- une diminution de durée de la courbe d'apprentissage et une meilleure reproductibilité des gestes;

- une invasivité chirurgicale moins délabrante ;

- une réduction de la durée d'intervention chirurgicale et/ou de la durée d'hospitalisation;

- un développement du maintien à domicile des patients.

Les exemples sont nombreux : les guides de coupe en chirurgie orthopédique, la chirurgie robotisée, la télésurveillance, les dispositifs d'hémostase et de suture mécanique ou encore, les dispositifs de contrôle de la profondeur de l'anesthésie...

Dans le domaine des systèmes d'information, les DM peuvent contribuer à améliorer le fonctionnement du système de soins. Leur évaluation doit alors intégrer des critères de processus (délais, coûts, facilité d'usage...).

Au regard des éléments ci-dessus, il apparaît que les critères d'évaluation des DM peuvent être de nature très différente en fonction des contextes d'usage.

Ainsi, l'ensemble des participants à la table ronde propose de retenir la définition suivante des critères cliniques : «Les critères cliniques sont les critères de morbi-mortalité. Sont aussi considérés comme critères cliniques les critères intermédiaires ou ayant un impact direct ou indirect sur la morbi-mortalité ». De manière conventionnelle, les critères cliniques correspondent aux objectifs thérapeutiques du traitement (la réduction de la morbi-mortalité) alors que les critères intermédiaires documentent plutôt les mécanismes d'action du traitement (des paramètres biologiques ou physiologiques). Les critères indirects, selon l'acception du terme par les participants à la table ronde, sont des mesures qui ne reflètent pas les mécanismes d'action du traitement mais en conditionnent le résultat, par exemple : l'observance et la persistance au traitement des patients. Logiquement, les participants à la table ronde proposent la définition suivante des critères non cliniques : "Les critères non cliniques englobent l'ensemble des autres critères. Ils peuvent avoir un impact individuel ou collectif. Ils concernent les patients, leur entourage, les soignants et les structures de soins, la collectivité dans son ensemble ». Cette définition met en exergue que les critères non cliniques ne se limitent pas au patient-related-outcomes (PRO). ${ }^{[1,2]}$ Cette définition signale que l'évaluation des DM (mais peut-être aussi de toute technologie de santé, médicaments inclus) doit s'attacher à considérer le point de vue de tous les acteurs du système de santé.

\section{Eléments de contexte}

Force est de constater que les critères actuellement pris en compte par les autorités de santé sont beaucoup plus restrictifs. Ainsi, dans son rapport d'activité de 2013, ${ }^{[3]}$ la CNEDiMTS précise que : "Le critère de jugement doit être cohérent avec l'effet thérapeutique ou de compensation du handicap annoncé. Ces critères retenus sont des critères cliniques (mortalité, morbidité, compensation du handicap, de qualité de vie, réduction des effets indésirables), ou de commodité d'emploi avec bénéfice clinique pour les patients ». Pour la CNEDiMTS, les critères cliniques incluent donc la mortalité, la morbidité, la compensation du handicap, la qualité de vie et la réduction des effets indésirables. Le critère de commodité d'emploi n'a pas de valeur en soi. Pourtant, tel que défini par le code de la Sécurité sociale (article R165-2), le service attendu (SA) évalué par la CNEDiMTS devrait l'être en fonction des deux critères suivants : d'une part l'intérêt du produit au regard de son effet thérapeutique, diagnostique ou de compensation du handicap et sa place dans la stratégie thérapeutique, d'autre part son intérêt de santé publique attendu (ISP). Or, dans sa définition réglementaire l'ISP comprend notamment l'impact sur la santé de la population, en termes de mortalité, de morbidité et de qualité de vie, la capacité à répondre à un besoin thérapeutique, diagnostique ou de compensation du handicap non couvert, eu égard à la gravité de la pathologie ou du handicap, l'impact sur le système de soins et l'impact sur les politiques et programmes de santé publique.

Aujourd'hui, la CNEDiMTS privilégie le seul point de vue du patient. A contrario, le core model de l'European Network for Health Technology Assessment (EUnetHTA) ${ }^{[4]}$ pour l'évaluation des technologies de santé adopte une perspective plus large, intégrant le point de vue de l'ensemble des acteurs du système de santé (les patients, mais aussi les professionnels de santé et la collectivité dans son ensemble). Au-delà des critères usuels d'efficacité et de sécurité, le core model d'EUnetHTA prend en compte de nombreuses autres préoccupations, à titre d'exemple : la santé des professionnels de santé (impact sur la sécurité au travail), les conditions d'usage de la technologie (impact sur les compétences requises, les besoins de formation, les processus d'apprentissage), le développement durable (impact sur l'environnement), les attentes des patients et de la population vis-à-vis de la technologie (acceptabilité, impact sur la participation sociale des patients et sur les inégalités sociales de santé), l'organisation des soins (impact sur les structures et les processus, impact sur les modalités de gestion). Le core model d'EUnetHTA constitue donc une référence utile pour l'évaluation multidimensionnelle des DM, même s'il ne précise pas encore à ce jour comment intégrer l'ensemble de ces dimensions pour arriver à un avis cohérent. Les modèles 
d'aide à la décision multicritères peuvent s'avérer utiles à cette fin. ${ }^{[5]}$

La connaissance des modèles de Health Technology Assessment (HTA) se diffuse progressivement dans les établissements de santé. ${ }^{[6,7]}$ Cette typologie d'évaluation est de plus en plus souvent mobilisée dans le cadre du référencement des technologies de santé par les Commissions des Médicaments et des Dispositifs Médicaux Stériles (COMEDIMS). ${ }^{[8]}$ L'expérience ancienne du Comité d'Evaluation et de Diffusion des Innovations Technologiques (CEDIT) à l'Assistance Publique - Hôpitaux de Paris (AP$\mathrm{HP}$ ) en la matière mérite d'être mise en exergue. Plus récemment, d'autres établissements ont mis en place des processus d'évaluation des technologies de santé du même type. Toutefois, ces modalités sont encore très hétérogènes d'un établissement à l'autre, même si des initiatives sont actuellement en cours pour standardiser ces procédures. Dans le cadre de certaines de ces procédures de référencement, les mesures de l'impact sur l'organisation des soins occupent naturellement une place importante puisque les établissements de santé supportent directement les coûts et les bénéfices induits par l'introduction des nouvelles technologies de santé.

L'introduction récente du critère médicoéconomique dans les procédures d'accès à la prise en charge par la collectivité des technologies de santé est aussi de nature à élargir la perspective de l'évaluation. Le décret 2012-1116 du 2 octobre 2012 relatif aux missions médicoéconomiques de la Haute Autorité de Santé (HAS) précise que l'évaluation doit prendre en compte non seulement le triptyque efficacité/qualité/sécurité, les coûts et la qualité de vie des patients mais aussi l'organisation des soins, les pratiques professionnelles, les conditions de vie des malades, l'amélioration de l'égal accès aux soins et le respect des règles éthiques. Le texte prévoit donc la prise en compte de critères autres que ceux définis par la CNEDiMTS dans son rapport d'activité. L'évaluation médicoéconomique, lorsqu'elle est conduite dans la perspective sociétale (conformément au guide méthodologique de la HAS), intègre le point de vue de l'ensemble des acteurs du système de santé à la fois dans la mesure des coûts (prise en compte des impacts organisationnels liés à l'introduction de la technologie, pertes de productivité) et dans la mesure des bénéfices (prise en compte des impacts sur la qualité de vie des patients et de leurs proches, introduction des critères d'équité). Le champ de l'évaluation peut être restreint en pratique pour des problèmes de mesure (par exemple, lorsqu'il s'agit de prendre en compte l'impact de la maladie sur la qualité de vie des proches ou lorsqu'il s'agit de mesurer les coûts indirects). Enfin, dans les dispositifs actuels, l'évaluation médicoéconomique est conduite en parallèle de l'évaluation médico-technique et ne contribue pas à la détermination du service attendu (SA) et de l'amélioration du service attendu (ASA). De plus, peu de DM répondent au critère de chiffre d'affaires conditionnant l'évaluation par la CEESP (20 millions d'euros TTC à deux ans) : à ce jour, sur près de trente avis d'efficience évalués ou en cours d'évaluation par la CEESP, un seul concerne un DM.

Sur la base des discussions précédentes, l'ensemble des participants à la table ronde reconnaissent l'importance d'intégrer des critères non cliniques à l'évaluation des DM.

\section{Comment intégrer les critères non cliniques dans l'évaluation des dispositifs médicaux?}

Les participants de la table ronde, après avoir validé l'intérêt de l'intégration de la dimension non clinique dans l'évaluation des DM, se sont accordés pour dire que cette dimension non clinique ne devait pas prévaloir, d'un point de vue méthodologique dans le cadre de l'évaluation, sur la dimension clinique. Il existe néanmoins certaines situations où la seule dimension pertinente dans le cadre de l'évaluation est la dimension non clinique. Dans ce cas, et dans ce cas seulement, la dimension non clinique prend le pas sur les critères cliniques d'évaluation.

On citera ici quelques exemples :

- les contraceptifs (médicamenteux ou DM) pour lesquels l'objectif n'est pas un objectif clinique (la grossesse n'est pas une maladie) mais bien un objectif sociétal, qui doit être valorisé comme tel ;

- les custom packs, qui regroupent l'ensemble des DM à usage unique, nécessaires pour une intervention chirurgicale, pouvant inclure ou pas de l'instrumentation à usage unique, et dont l'alternative est la préparation extemporanée de chaque DM utilisé lors de l'intervention; il n'y a pas ici d'objectif clinique direct pour le patient, mais bien un objectif organisationnel et économique à évaluer ;

- la télémédecine qui conjugue des impacts cliniques et non cliniqes.

En outre, il a été plusieurs fois précisé lors des discussions que l'évaluation de la dimension non clinique ne pouvait en aucun cas se soustraire à la rigueur méthodologique d'évaluation qui prévaut pour l'évaluation clinique des DM.

Ainsi, la dimension non clinique est :

- soit la seule pertinente qui doit être évaluée avec la même rigueur que la dimension clinique, c'est-à-dire être le critère primaire d'une étude méthodologiquement bien conduite ;

- soit une dimension complémentaire à la dimension clinique, permettant d'apporter un éclairage nouveau, et devant toujours faire l'objet d'une réelle évaluation. Dans ce cas, le ou les critères non cliniques peuvent être des critères secondaires d'une 


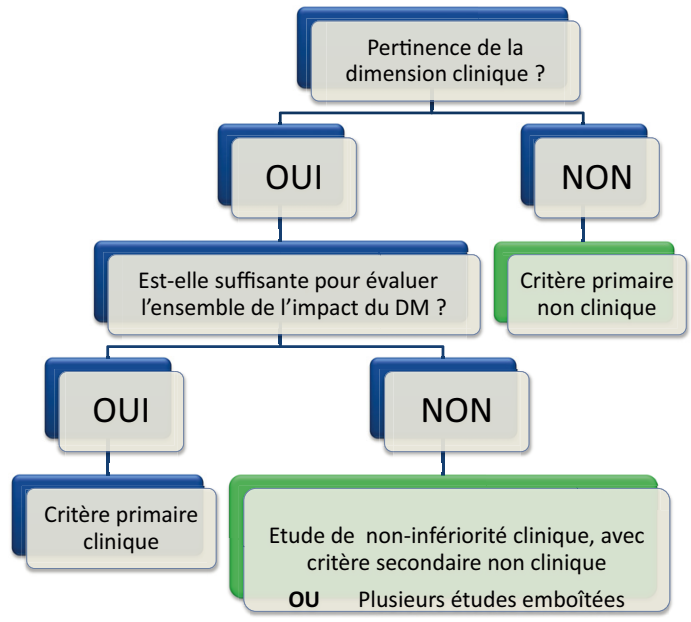

Fig. 1. Arbre décisionnel pour l'évaluation des dispositifs médicaux. DM : dispositifs médicaux.

étude ayant pour critère primaire un critère clinique (étude de supériorité ou de non-infériorité).

De ces considérations est ressorti un arbre décisionnel pour l'évaluation des DM, qui permet de donner sa place à la dimension non clinique dans l'évaluation (figure 1).

Le point positif concernant la possibilité de l'intégration de la dimension non clinique dans l'évaluation du DM en France est que l'ensemble des outils sont déjà disponibles, comme présenté dans la partie précédente : par exemple les modèles EUnetHTA, Evidence and Value : Impact on Decision Making (EVIDEM) ${ }^{[9]}$ et Model for Assessment of Telemedicine (MAST) intègrent des éléments organisationnels, éthiques, culturels. Une attention toute particulière devra être portée à la transposition culturelle et linguistique de ces outils en France, qui sera d'ailleurs peut-être différente en fonction des objectifs recherchés (admission au remboursement au niveau national ou adoption au niveau local dans un établissement).

De la même manière, même s'il a été précisé que la méthodologie d'étude mise en œuvre doit être aussi rigoureuse que pour les objectifs cliniques, le groupe reste conscient du fait que les critères non cliniques sont directement liés à l'environnement et qu'ils doivent donc souvent être mesurés directement dans l'environnement d'étude. Ainsi la question de la transposabilité contextuelle des résultats, d'un pays à l'autre, voire d'un établissement à l'autre, se pose avec plus d'acuité que dans le cas des critères cliniques. Ceci rend plus compliquée et plus coûteuse la mise en place d'études sur les aspects organisationnels, culturels, éthiques....

Le design de l'étude devra, autant que faire se peut, en fonction des contraintes budgétaires et de déploiement, prendre en compte l'ensemble de ces éléments contextuels de façon à répondre au mieux à la question posée.

L'ensemble de ces questions de méthodologie et de transposabilité des résultats pourra faire l'objet d'une réflexion spécifique ultérieure, les participants de la table ronde n'ayant pas eu le temps lors des ateliers de se pencher plus avant sur ces questions.

\section{Intégrer les critères non cliniques pour contribuer à une meilleure évaluation des $\mathrm{DM}$ et des produits de santé}

Un consensus fort s'est établi entre les participants de la table ronde sur le besoin d'intégrer les critères non cliniques dans l'évaluation des DM pour une meilleure évaluation et une meilleure prise de décision au niveau national comme au niveau local.

En outre, comme on l'a vu précédemment, certains outils d'évaluation existent déjà, et les textes réglementaires pour l'évaluation au niveau national permettent déjà d'intégrer la dimension non clinique.

L'arbre de décision proposé pour le choix des critères (clinique ou non clinique) dans l'évaluation des DM peut servir de base à des travaux complémentaires et de structure pour l'implémentation effective de ces outils. Néanmoins de nombreuses questions restent aujourd'hui non résolues et méritent d'être étudiées de près.

La question de la transposabilité des résultats des études a déjà été soulevée dans la section précédente. Pour rester dans la méthodologie des études, le choix des critères non cliniques pertinents en fonction des domaines (DM implantables, maladies chroniques, handicap...) pourra être étudié en détail de façon à proposer aux industriels des « guides » de choix de critères pertinents en fonction des typologies de DM.

Une fois la pertinence et la faisabilité de l'évaluation non clinique étudiées, vient la problématique de l'intégration dans la prise de décision. Au niveau national, les textes réglementaires prévoient déjà l'intégration de ces critères dans l'évaluation soit au travers de l'évaluation par la CNEDiMTS dans le cadre de l'intérêt de santé publique (ISP) ${ }^{2}$, soit dans le cadre de l'évaluation médicoéconomique par la CEESP ( $c f .2$ ).

Force est de constater aujourd'hui que l'ISP est peu, voire pas évalué ce qui pose la question de la légitimité de son évaluation par la CNEDiMTS, ou de sa refonte totale, de façon à intégrer des critères complémentaires et à prévoir l'articulation avec l'évaluation médicoéconomique le cas échéant.

\footnotetext{
${ }^{2}$ Pour rappel le service attendu est un critère composite qui intègre notamment l'appréciation de l'efficacité et des risques, de la place du DM évalué dans la stratégie de prise en charge et de son impact sur la santé publique (ISP).
} 
Il est important de prévoir les modalités pratiques de l'évaluation car elles auront un impact direct sur la prise de décision. Les questions qui devront être posées et débattues sont les suivantes : à qui incombe la responsabilité de l'évaluation des critères non cliniques? Comment ces critères non cliniques peuvent-ils être pondérés par rapport aux critères cliniques et en fonction des objectifs de santé publique ? Le régulateur est-il prêt à accepter une baisse de l'apport clinique «compensée » par un apport sur une dimension non clinique, dans quelle mesure, quelle doit en être la contrepartie? Qu'en est-il du payeur? Doit-on envisager l'intervention de payeurs autres que l'Assurance Maladie, telles les complémentaires santé, les collectivités territoriales, la Caisse Nationale de Solidarité pour l'Autonomie...?

Le DM a un rôle structurant de l'organisation des soins, tout particulièrement si on envisage le parcours patient dans son ensemble (télémédecine, implants orthopédiques pour chirurgie mini-invasive ayant un impact sur la durée du séjour hospitalier et la prise en charge en termes de rééducation...). Mais, dans un système dans lequel les enveloppes sont non fongibles, il est difficile de valoriser justement l'apport d'un changement d'organisation de l'hôpital vers la ville par exemple. Comment le régulateur et le payeur sont-ils prêts à valoriser l'innovation non clinique aujourd'hui?

C'est bien sûr l'un des éléments qui conditionnera la capacité des industriels à investir dans des études qui incluent la mesure de critères non cliniques, d'autant plus que ces variables risquent d'être non transposables d'un pays à l'autre. Les investissements liés à ces études risquent donc d'être importants et les industriels présents à la table ronde se sont interrogés sur l'accompagnement possible par les pouvoirs publics au financement de ces études.

Enfin, et pour conclure, il a été noté qu'un grand nombre de réflexions menées dans le cadre de cette table ronde pourraient être applicables à d'autres produits de santé dont certains peuvent également avoir un rôle structurant dans l'organisation des soins. Il serait donc intéressant d'ouvrir le champ de la réflexion de l'évaluation non clinique à l'ensemble des produits de santé.

Conflits d'intérêt. Sept participants à la table ronde sont en lien avec des sociétés commercialisant des DM (KS, AJ, KC, FD, OG, $\mathrm{PM}, \mathrm{CR}$ ). Trois participants appartiennent à des institutions ou commissions en charge de l'évaluation des DM (BD, CRP, JPS). Les participants à la table ronde ne déclarent pas de conflits d'intérêt particuliers en rapport avec cet article.
Abréviations. ASA : amélioration du service attendu; AP-HP : Assistance Publique-Hôpitaux de Paris ; CEDIT : Comité d'Evaluation et de Diffusion des Innovations Technologiques; CEESP : Commission d'Evaluation Economique et de Santé Publique; CNEDiMTS : Commission Nationale d'Evaluation des Dispositifs Médicaux et des Technologies de Santé; COMEDIMS : Commissions des Médicaments et des Dispositifs Médicaux Stériles; DM : dispositif médical; EUnetHTA : European Network for Health Technology Assessment ; EVIDEM : Evidence and Value: Impact on Decision Making ; HAS : Haute Autorité de Santé ; HTA : Health Technology Assessment; ISP : intérêt de santé publique; MAST : Model for ASsessment of Telemedicine; PRO : patient-related-outcomes; SA : service attendu.

\section{Références}

1. Patrick DL, Burke LB, Powers JH, et al. Patient-reported outcomes to support medical product labeling claims: FDA perspective. Value in Health 2007; nov-dec 10 (suppl 2): S125-37

2. Burke LB, Kennedy DL, Miskala PH, et al. The use of patient-reported outcome measures in the evaluation of medical products for regulatory approval. Clin Pharmacol Ther 2008 84(2): 281-3

3. Commission Nationale d'Evaluation des Dispositifs Médicaux et des Technologies de Santé (CNEDiMTS). Rapport d'activité 2013. Haute autorité de santé

http: //www . has-sante. fr Consulté le 6 janvier 2015 (124 pages)

4. European Network for Health Technology Assessment (EUnetHTA). The HTA Core Model $^{\circledR}$ Version 2.0. EUnetHTA Work Package 8, 28 Nov 2013 http://meka.thl.fi/htacore/model/HTACoreModel2.0.pdf Consulté le 6 janvier 2015 (357 pages)

5. Thokala P, Duenas A. Multiple criteria decision analysis for health technology assessment. Value Health 2012; 15(8): 1172-81

6. Gagnon MP, Desmartis M, Poder T, et al. Effects and repercussions of local/hospital-based health technology assessment (HTA): a systematic review. Syst Rev 2014; 3: 129

7. Gagnon MP. Hospital-based health technology assessment: developments to date. Pharmacoeconomics 2014; 32(9): 819-24

8. Martelli N, van den Brink H, Denies F, et al. Évaluation des technologies de santé en milieu hospitalier : quelle organisation pour évaluer et acquérir des dispositifs médicaux innovants. Ann Pharm Fr 2014; 72(1): 3-14

9. EVIDEM. Evidence and Value: Impact on Decision Making https: //www.evidem.org/

Consulté le 6 janvier 2015

Correspondance et offprints : Karine Szwarcensztein, Johnson \& Johnson Medical Companies France, Ethicon SAS, 1 rue Camille Desmoulins - TSA 81 002, 92787 Issy les Moulineaux Cedex 9, France.

E-mail : kszwarce@its.jnj.com 